\title{
Implications of diameter and volume-based measurement in assessment criteria for liver transplantation for hepatocellular carcinoma
}

\author{
Alice I. Graham ${ }^{1}$, Anya Adair ${ }^{1}$, Ewen M. Harrison'1, James Gordon-Smith², Hamish Ireland², Dilip Patel², \\ Stephen J. Wigmore ${ }^{1}$
}

'Department of Clinical Surgery, University of Edinburgh, Edinburgh EH16 4SA, United Kingdom.

${ }^{2}$ Department of Radiology, Royal Infirmary of Edinburgh, Edinburgh EH16 4SA, United Kingdom.

Correspondence to: Prof. Stephen J. Wigmore, Department of Surgery, Royal Infirmary of Edinburgh, Royal Infirmary of Edinburgh 51 Little France Crescent Edinburgh EH16 4SA, Edinburgh EH164SA, United Kingdom. E-mail: wigmore@ed.ac.uk

How to cite this article: Graham Al, Adair A, Harrison EM, Gordon-Smith J, Ireland H, Patel D, Wigmore SJ. Implications of diameter and volume-based measurement in assessment criteria for liver transplantation for hepatocellular carcinoma. Hepatoma Res 2021;7:1. http://dx.doi.org/10.20517/2394-5079.2020.87

Received: 21 Aug 2020 First Decision: 10 Oct 2020 Revised: 26 Oct 2020 Accepted: 1 Dec 2020 Published: 7 Jan 2021

Academic Editor: Geoff McCaughan, James Fung Copy Editor: Monica Wang Production Editor: Jing Yu

\begin{abstract}
Aim: Eligibility for liver transplantation for hepatocellular carcinoma (HCC) is currently based on singledimension, diameter measurements on cross-sectional imaging, as specified by various selection criteria. This does not account for significant differences in shape, and therefore tumour volume, between patients. This study investigated whether one-dimensional selection criteria disadvantages patients by not considering volume.
\end{abstract}

Methods: Patient data were collected retrospectively from a prospectively maintained database. Tumours were measured on both computer tomography (CT) and magnetic resonance imaging (MRI). Tumour volume was measured using two methods; semi-automated planimetry and the ellipsoid volume formula. Statistical analysis was performed using SPSS.

Results: A total of 313 patients with HCC were assessed for liver transplantation. For this study, patients who underwent transplantation $(n=89)$ and those who did not based on tumour size $(n=33)$ were included. In total, 213 tumours were measured, showing excellent correlation between CT and MRI $\left(R^{2}=0.83\right)$. The majority of tumour nodules (94\%) were ellipsoid not spherical. Volumetric measurements of the 84 tumours that did not meet diameter-based Milan criteria confirmed that $76 \%$ would have been within a theoretical volume allowance based on Milan criteria diameters.

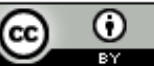

(C) The Author(s) 2021. Open Access This article is licensed under a Creative Commons Attribution 4.0 International License (https://creativecommons.org/licenses/by/4.0/), which permits unrestricted use, sharing, adaptation, distribution and reproduction in any medium or format, for any purpose, even commercially, as long as you give appropriate credit to the original author(s) and the source, provide a link to the Creative Commons license, and indicate if changes were made. 
Conclusion: This study shows that a significant number of patients deemed outside conventional diameter-based Milan criteria have smaller tumour volumes than those considered within criteria. It appears that those with ellipsoid rather than spherical tumours may be disadvantaged by current size-based criteria. Further research using a contemporary patient cohort who have had the benefit of advancements in non-surgical treatments for $\mathrm{HCC}$ is required.

Keywords: Hepatocellular carcinoma, liver transplantation, Milan criteria, HCC measurement

\section{INTRODUCTION}

Worldwide, hepatocellular carcinoma (HCC) is the 4th highest cause of cancer-related mortality and the 6 th most prevalent cancer ${ }^{[1]}$. A wide variety of diseases are well recognised as risk factors for the development of HCC, which carries a significant burden of cancer-related disease throughout the world ${ }^{[2]}$. An estimated $80 \%$ of cases in the developing world are a result of chronic infection with hepatitis B virus (HBV). In contrast, the biggest risk factors in the developed world are alcohol and hepatitis $\mathrm{C}$ virus infection $(\mathrm{HCV})^{[3]}$. Any patient with cirrhosis is at risk of developing HCC and other risk factors include diabetes, obesity and smoking ${ }^{[4]}$.

Diagnosis of HCC can be made by radiological imaging of a nodule which "is defined by intense contrast uptake during the arterial phase, followed by contrast washout during venous or delayed phases in a contrast-enhanced study ${ }^{\text {"[3] }}$. This is usually by computer tomography (CT) or magnetic resonance imaging (MRI) which has a specificity of $\sim 100 \%$ and a sensitivity $>70 \%{ }^{[3,5]}$. CT is widely available, quick to perform and the most commonly used imaging modality for $\mathrm{HCC}^{[6]}$. However, it has the disadvantages of a high radiation dosage and relative insensitivity compared with MRI, with some benign lesions often mistaken for tumours ${ }^{[6]}$. Recent advances in technology, including improved resolution and the use of contrast agents, have greatly increased MRI specificity and sensitivity for $\mathrm{HCC}^{[6]}$.

Diagnosis of early HCC increases 5-year survival to $>50 \%$, with curative treatment options including ablation, resection and orthotopic liver transplantation $(\mathrm{OLT})^{[3,7,8]}$. The last is widely accepted as the best treatment both in practice and theory, as it gives the widest resection margins, removes the risk of de novo cancer development in the remaining diseased liver and allows restoration of hepatic function ${ }^{[9,10]}$. One of the biggest limiting factors to OLT is donor shortage, although this has been partly addressed now by live donor transplantation ${ }^{[3,9]}$. To ensure the livers go to those who will benefit most, OLT is currently only offered if patients meet certain eligibility criteria.

Before the advent of the Milan criteria in the mid-1990s, the broader OLT selection criteria for HCC resulted in high levels of cancer recurrence (32\%-54\%) and poor survival (<40\% at 5 years) ${ }^{[7]}$. However, during this time extensive research was conducted on the characteristics of the patients with favourable outcomes post-transplantation $^{[7]}$. With these results, Mazzaferro published the Milan criteria in determining eligibility for OLT ${ }^{[1]}$. These criteria were widely adopted and suggested that only patients with a solitary HCC nodule with a maximum diameter of $5 \mathrm{~cm}$ or up to three lesions, each less than $3 \mathrm{~cm}$, should be eligible for transplantation. Application of these criteria increased 5-year survival in liver transplant centres to $70 \%$, with recurrence in $<15 \%$ of patients ${ }^{[7]}$. Since then, several alternative criteria have been proposed, with a wide evidence base suggesting that extending the criteria has little effect on long-term survival ${ }^{[12]}$. As such, the UK liver transplant community has adopted an extended form of the Milan criteria increasing the number of tumours less than $3 \mathrm{~cm}$ from 3 to $5^{[13]}$ and permitting the transplantation of patients with single tumours between $5 \mathrm{~cm}$ and $7 \mathrm{~cm}$, where there is either a reduction in size of the tumour or stable tumour size on RECIST criteria ${ }^{[11]}$ after 6 months with or without treatment such as transarterial chemoembolisation. 
Current eligibility criteria for OLT only require the longest diameter measurement of tumours in one plane, historically the transverse/axial plane, by CT and MRI. However, with advances in technology, it is possible to measure lesions in 3 dimensions (3D), thereby enabling the calculation of total tumour volume (TTV). There is no current requirement under the Milan criteria for tumours to be evaluated in all planes to find the largest diameter. Data from studies in patients with pancreatic adenocarcinoma ${ }^{[12]}$ and colorectal carcinoma ${ }^{[13]}$ have shown that measurements in only one plane overestimate tumour burden in comparison to $3 \mathrm{D}$ measurement. Many now believe that volumetric assessment is superior to conventional techniques in representing tumour burden ${ }^{[14]}$. Tumour volume in HCC has already been shown to be a superior predictor of survival in patients undergoing transarterial chemoembolisation than diameter-based measurements ${ }^{[14]}$. Although there are published studies investigating the relationship between TTV and prognosis, many of these continue to assume that tumours are spherical ${ }^{[15,16]}$. However, measuring in $3 \mathrm{D}$ can theoretically increase any potential errors three-fold ${ }^{[15]}$. Moreover, with the use of volumetric software programmes (also known as planimetry), this process can become semi-automatic, decreasing human variation. Furthermore, there are currently no studies comparing the accuracy of planimetry to using the standard formula for ellipsoid volume.

Evidence-based treatment of HCC is still very limited, with $<100$ randomised control trials published ${ }^{[7]}$. Despite the fact that eligibility for OLT is dependent on tumour size, assessed by radiological imaging, there is little evidence on its accuracy. There is also limited research comparing diameter to volume in determining eligibility for OLT and its impact on the ultimate survival of patients. This study aimed to investigate these issues and hypothesised that HCCs are not spherical, as assumed by current criteria. We also predicted that current diameter-based measurements alone would disadvantage patients whose tumours are not spherical. We hypothesised that volume measurements would better represent true tumour burden and are therefore more appropriate when assessing HCC for OLT.

\section{METHODS}

\section{Patients}

Patients were selected for this study from the Scottish Liver Transplant Unit (SLTU) database, based at the Royal Infirmary of Edinburgh, which provides services to the whole of Scotland. Since its establishment in 1992, over 1,600 transplants have been performed. Baseline information on patient demographics and aetiology of underlying liver disease was extracted and supplemented with information from NHS Lothian's patient database, TRAK. The SLTU currently uses the extended Milan criteria (one tumour $<5 \mathrm{~cm}$ or 5 tumours $\leq 3 \mathrm{~cm})^{[13]}$. The diagnosis and definition of HCC was based on RECIST guidelines ${ }^{[11]}$.

\section{Imaging and measurement of tumours using the Milan criteria}

Tumours of patients who were transplanted $(n=129)$ and those who were considered outside the Milan criteria $(n=84)$ were identified from the SLTU database. The tumour images closest to the STLU assessment date were selected. All images were viewed and measured at the same magnification on the radiology software package (PACS, Carestream Health) used by NHS Lothian. Tumours were measured in $\mathrm{mm}$ on both CT and MRI where possible, with the maximum allowed interval time between scans being 12 weeks. The measurement recorded was the largest diameter on transverse section using callipers and the measuring function. The same scans were used to measure both diameter and volume. The explant pathology only provided the largest diameter of the tumours and was not available for all patients.

All tumour measurements were made by the same author throughout the study (AG). However, 2 additional authors (SW and AA) separately measured a sample of 10 tumours to assess inter-user variability and examine the effect of the magnification of the image on the accuracy of the measurements. A protocol was created to ensure that all measurements were similarly performed [Appendix 1]. 
Table 1.Diagnoses of patients with HCC assessed by SLTU

\begin{tabular}{lcc}
\hline Diagnosis & No. of patients & \% of patients \\
\hline Alcoholic liver disease & 123 & 39.5 \\
Hepatitis C & 116 & 37.3 \\
Non-alcoholic fatty liver disease & 33 & 10.6 \\
Haemachromatosis & 20 & 6.4 \\
Hepatitis B & 18 & 5.8 \\
Primary biliary cirrhosis & 18 & 5.8 \\
Autoimmune hepatitis & 10 & 3.2 \\
Cholangiocarcinoma & 9 & 2.9 \\
Haemophilia & 8 & 2.6 \\
Primary sclerosing cholangitis & 5 & 1.6 \\
HIV & 4 & 1.3 \\
Budd Chiari & 1 & 0.3 \\
Carcinoid tumour & 1 & 0.3 \\
\hline
\end{tabular}

HCC: hepatocellular carcinoma; SLTU: the Scottish Liver Transplant Unit; HIV: human immunodeficiency virus

The diameter based Milan criteria and their volume equivalents were defined as a solitary HCC $\leq 5 \mathrm{~cm}$ in diameter (equivalent to total tumour volume TTV $\leq 65.5 \mathrm{~mL}$ ) or $\leq 5$ lesions, each $\leq 3 \mathrm{~cm}$ (equivalent to $\mathrm{TTV} \leq 14.1 \mathrm{~mL}$ each). To calculate tumour volume, two methods were used: first, using the equation for ellipsoid volume $4 / 3 \pi(a / 2+b / 2+c / 2)$ where $a, b$ and $c$ are the diameters of the tumour, measured in 3 perpendicular planes, and second, using volumetric software. The latter involved the user drawing round tumour outlines on each slice of radiology using a freeform contouring tool. The TTV was calculated for each patient by summing individual tumour volumes. For volume comparisons, MRI scans were used.

\section{Statistical analysis}

Data analysis and statistical tests were carried out using Microsoft Excel and IBM SPSS. Student's $t$-test was used for comparison of paired variables across patient groups. Patient survival was calculated until death or the time of study (30/11/2013). Kaplan-Meier analysis was used for survival of patients. A $P$ value of $<0.05$ was considered statistically significant. These statistical methods were based on those used previously in similar studies ${ }^{[12,16,17]}$.

\section{RESULTS}

\section{Patient characteristics}

The STLU database contained 313 patients with HCC who were assessed between 1994 and 2013. The median age of the patients was 60 years (range 14-75), including 244 males and 67 females. There was a wide variety of underlying diagnoses, the most common of which was alcoholic liver disease (40\%), followed closely by HCV [Table 1].

One hundred sixty-eight patients were listed for transplant and 143 were not [Figure 1]. In the current study, tumours were measured in those with available imaging in 2 patient groups; those eligible $(n=89)$ or not $(n=33)$ for OLT, based solely on the diameter of their tumours and the expanded Milan criteria. These patient groups had 129 and 84 tumours, respectively, which were measured in one dimension on both CT and MRI. Volume was calculated from patients with MRI images ( $n=63$ and $n=46$, respectively). The other patient groups $(n=95)$ were excluded due to the presence of variable risk factors such as recidivism, comorbidities and disease progression.

The average diameter of the tumours was $23.2 \pm 11.5 \mathrm{~mm}$ [mean \pm standard deviation (SD)]. $58 \%$ of patients had one HCC, $33 \%$ had $\leq 3$ and the remaining $9 \%$ of patients had $>3$. 


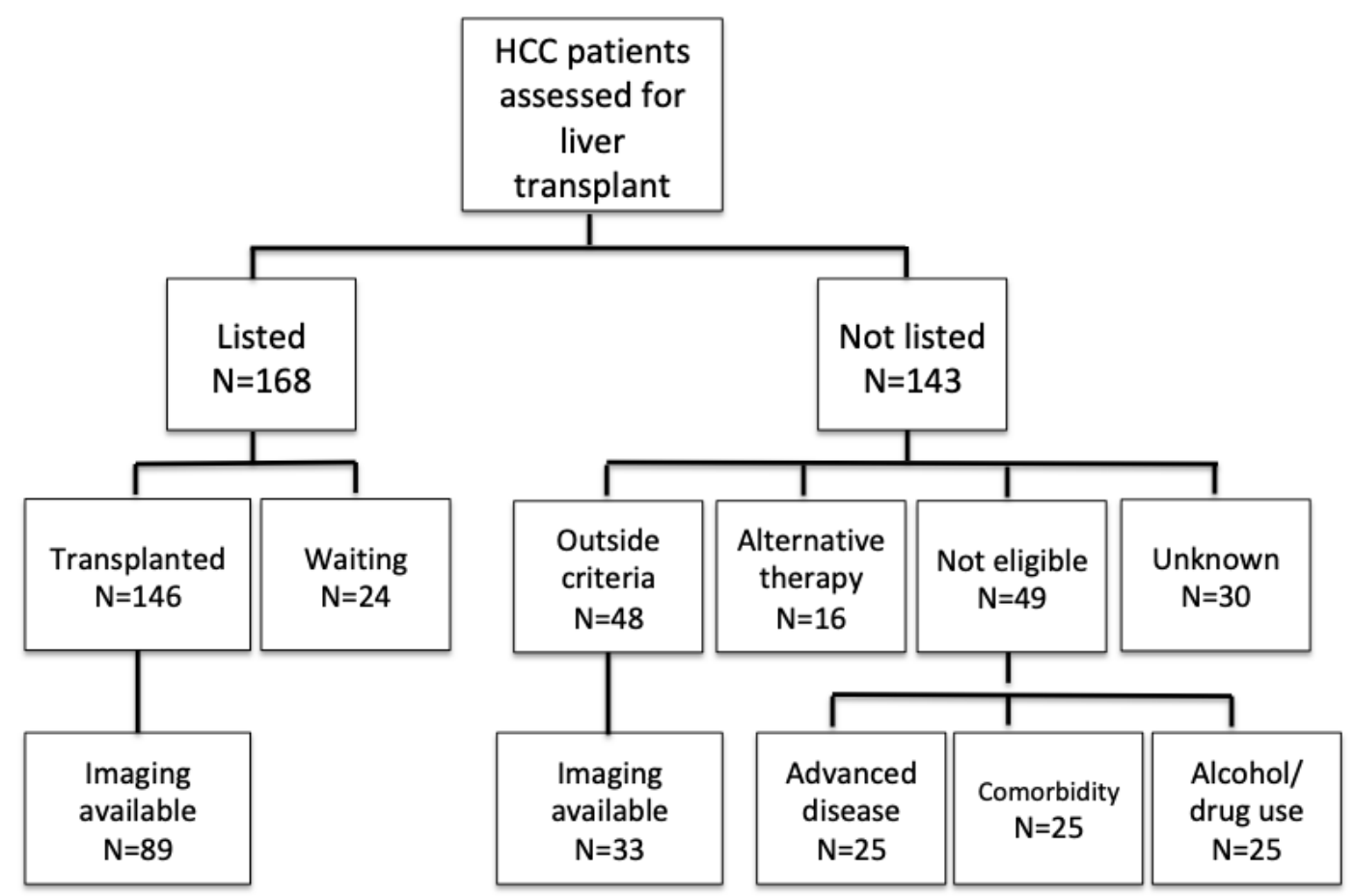

Figure 1. Outcomes and availability of imaging in patients with HCC on the SLTU database. HCC: hepatocellular carcinoma; SLTU: the Scottish Liver Transplant Unit

\section{Diameter-based criteria}

To assess the validity of current diameter-based OLT eligibility criteria for HCC, a number of variables were investigated. Firstly, we compared the measurements of tumour diameter on CT and MRI, as the current criteria does not specify the use of one method over the other. This study found no statistically significant difference $(P=0.536)$ between the two imaging modalities [Figure $2 \mathrm{~A}]$.

Secondly, radiological measurements of tumours, for both CT $(n=118)$ and MRI $(n=112)$, were compared to pathology reports [Figure $2 \mathrm{~B}$ ]. Radiology tended to overestimate the size of small tumours and underestimate the larger nodules, compared to the pathology reports. The difference between CT and pathology was significant $(P=0.014)$ but between MRI and pathology was not $(P=0.094)$.

Finally, we examined the effect of inter-user variability and magnification on the measurement of tumours, since HCCs that are assessed for transplantation may be measured by numerous radiologists and are potentially subject to error between users. The Milan criteria do not specify the magnification at which tumours should be measured. Our research, in which 3 independent authors measured a sample of 10 lesions, showed no statistically significant difference in the percentage standard deviation of measurements at different magnifications between users [Figure 2C].

\section{Shape of HCCs}

Mathematical 3D models were created to demonstrate variability between diameter and corresponding volume. This showed how tumours with the same diameters (and same dimension on a transverse section on radiology) may have very different TTVs. Figure 3 shows how stretching a sphere in different dimensions alters the volume as well as the shape. Despite increasing the y dimension from 3 to $4 \mathrm{~cm}$ between shape A and $\mathrm{E}$, the volume of the corresponding ellipsoid actually decreases from $14.1 \mathrm{~mL}$ to $12.6 \mathrm{~mL}$. This demonstrates how a tumour that appears to be larger on radiology (i.e., in one dimension) than another tumour may really have a smaller TTV. 

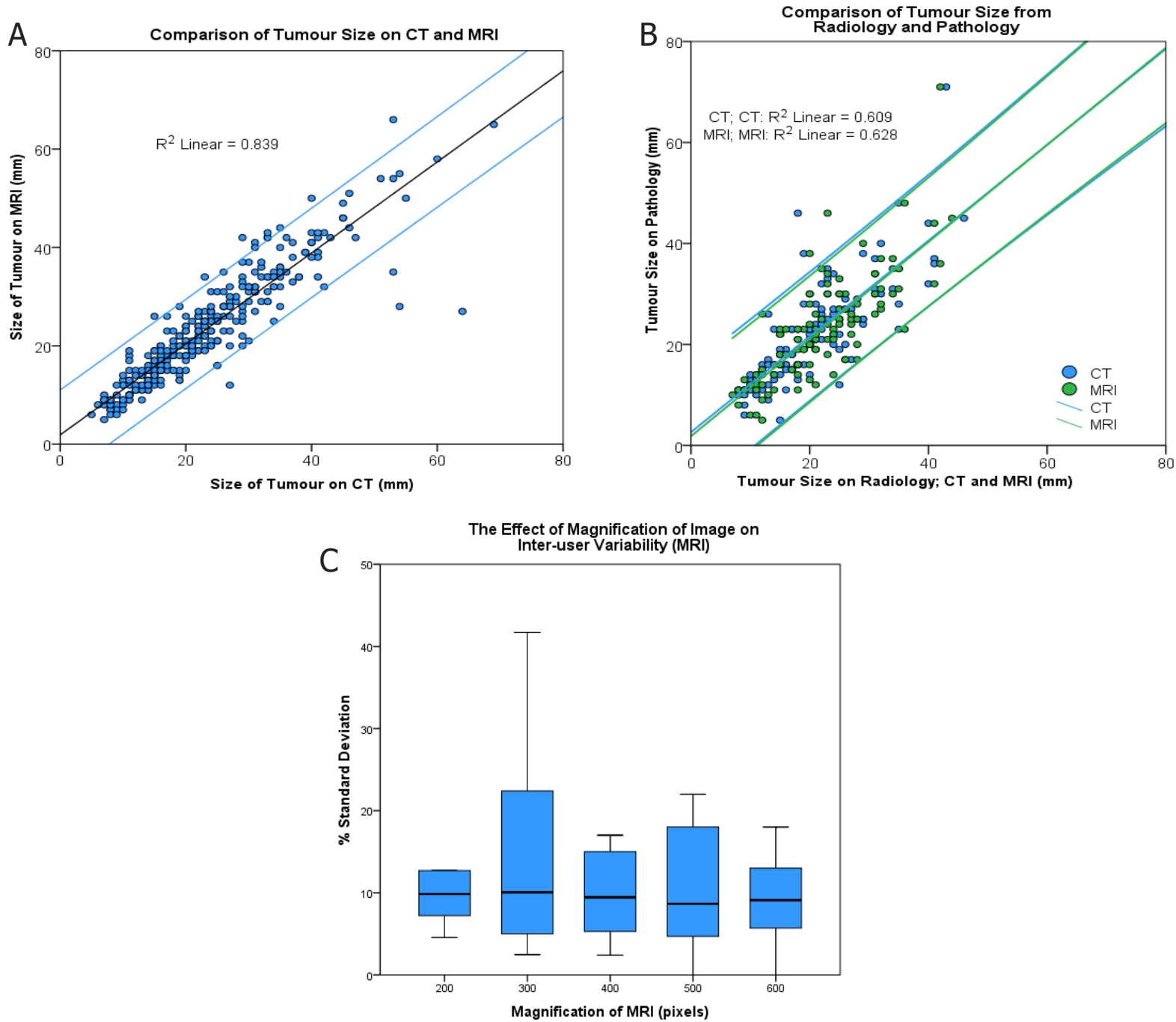

Figure 2. Comparison of tumour size on $C T$ and MRI. Student's $t$-test was conducted to compare measurements $[t(310)=-0.620, P$ $=0.536](A)$; comparison of tumour sizes measured by radiology and pathology. Student's t-test was again conducted to compare the 2 paired samples for $C T[t(117)=2.494, P=0.014]$ and $M R I ~[t(111)=1.689, P=0.094]$ (B); the effect of magnification on interuser variability of tumour measurements. The graph shows the percentage standard deviation of tumour measurements between 3 independent raters at 5 different magnifications. There was no statistically significant difference $(P>0.05)(C)$; CT: computer tomography; MRI: magnetic resonance imaging

This relationship was found to apply in practice to the tumours measured in this study: $94 \%$ were ellipsoid in shape, even when allowing for $5 \%$ deviation between the perpendicular diameters. Figure $4 \mathrm{~A}$ shows a patient's tumour that would have very different "maximum diameters" depending on the plane of measurement used.

This relationship between shape and volume can be further presented by comparing the true TTV of patients to the volume if the tumours are assumed to be spherical based on its diameter (Figure $4 \mathrm{~B}, P=$ 0.015). It shows that, by assuming all tumours are spherical, volume will be grossly over-estimated.

\section{Volume-based criteria}

As noted earlier, there are two recognised methods for calculating the volume of tumours. First, the mathematical equation for the volume of an ellipsoid and 3 perpendicular diameters was used, and second, semi-automated planimetry software with a freeform contouring tool was used. A comparison of these is 

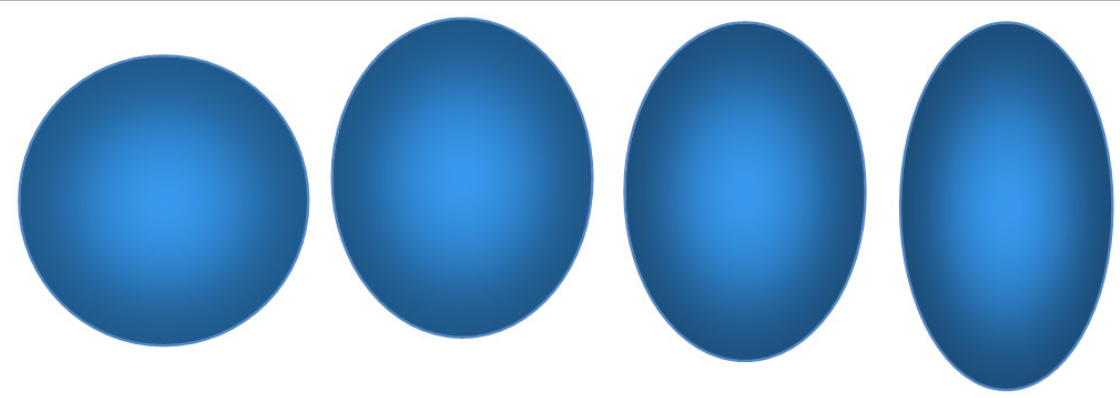

A

5.0

5.0

5.0

65.5
B

5.0

5.3

4.7

65.2
C

5.0

5.5

4.5

64.8

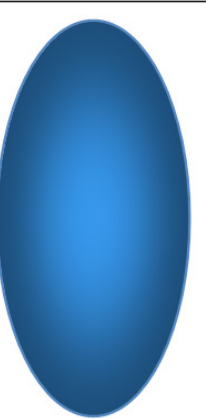

E

5.0

6.0

4.0

62.8

Total Tumou

Figure 3. Theoretical volume calculations based on ellipsoids of different dimensions based around the upper limit for the Milan criteria for a single tumour measuring $5 \mathrm{~cm}$ in maximum dimension

shown in Figure 4C. For the remainder of the study the planimetry software was used as it is assumed to be more accurate, allowing for variation in tumour circumference. Of the 109 patients whose TTV was calculated, all but one were within the volume criteria.

\section{Implications of research on the number of patients eligible for transplantation}

Table 2 shows that $95 \%$ of patients assessed were eligible for liver transplantation based on a volume-based interpretation of Milan criteria, but only 58\% were eligible based on conventional diameter-based Milan criteria. Only 57\% of patients met both criteria, but 38\% fell within volume but outside diameter criteria. According to this study, many more patients would be eligible for transplantation if the criteria were based on TTV rather than maximum tumour diameter. Figure 4D shows the overall survival for all patients who had tumour volumes within a volume-based approach to the extended Milan criteria based on whether they were also considered in or outside of Milan criteria based on conventional diameter-based assessment.

\section{Transplantation}

This study identified many patients who were eligible for OLT based on volume rather than diameter. OLT is considered to be the best curative option for $\mathrm{HCC}^{[9,10]}$. This is supported by this research as, when only the patients within theoretical volume-based criteria are considered, those who underwent transplantation (and therefore within diameter-based criteria) had a significantly higher 5-year survival [Figure 4D]. Whilst this survival difference reflects the benefit of transplantation, these patients are those with very similar-sized TTV, and therefore, exclusion based solely on size criteria has a large impact on survival. This is despite the exposure of transplanted patients to long-term immunosuppression and the risks of major surgery ${ }^{[18]}$. However, there is a selection bias between these two patient groups, as those who undergo transplantation will have smaller tumours which are eligible for OLT, a confounding factor that was unaccounted for in our survival analysis. It is also important to note that advancements in standard care for patients with HCC mean that very few, even those not listed for transplantation, receive no treatment at all. Our study did not aim to evaluate the benefits of non-surgical therapy. This analysis also included deaths of all causes, whether they were associated with HCC or not. Encouragingly, the 5-year survival for patients after transplantation at SLTU is higher than the $70 \%$ expected at tertiary centres ${ }^{[7]}$. 
A

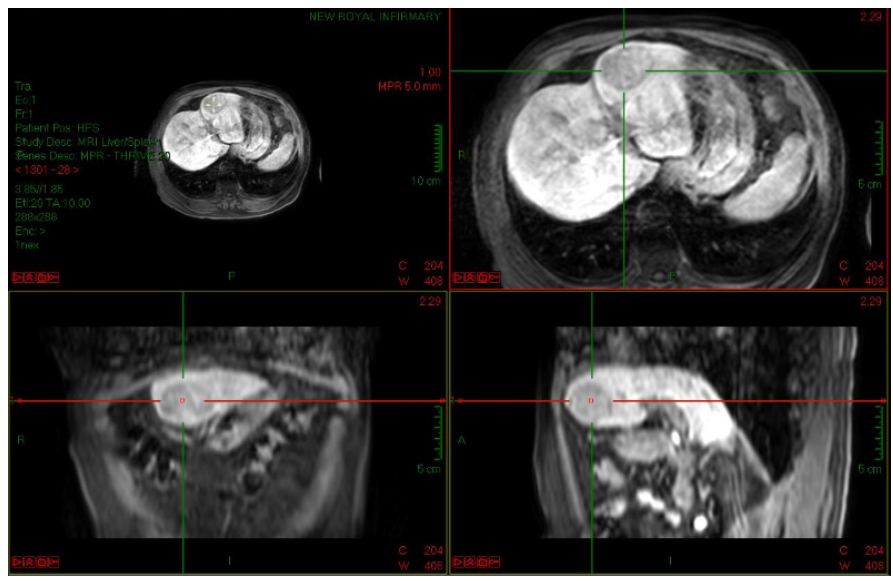

B
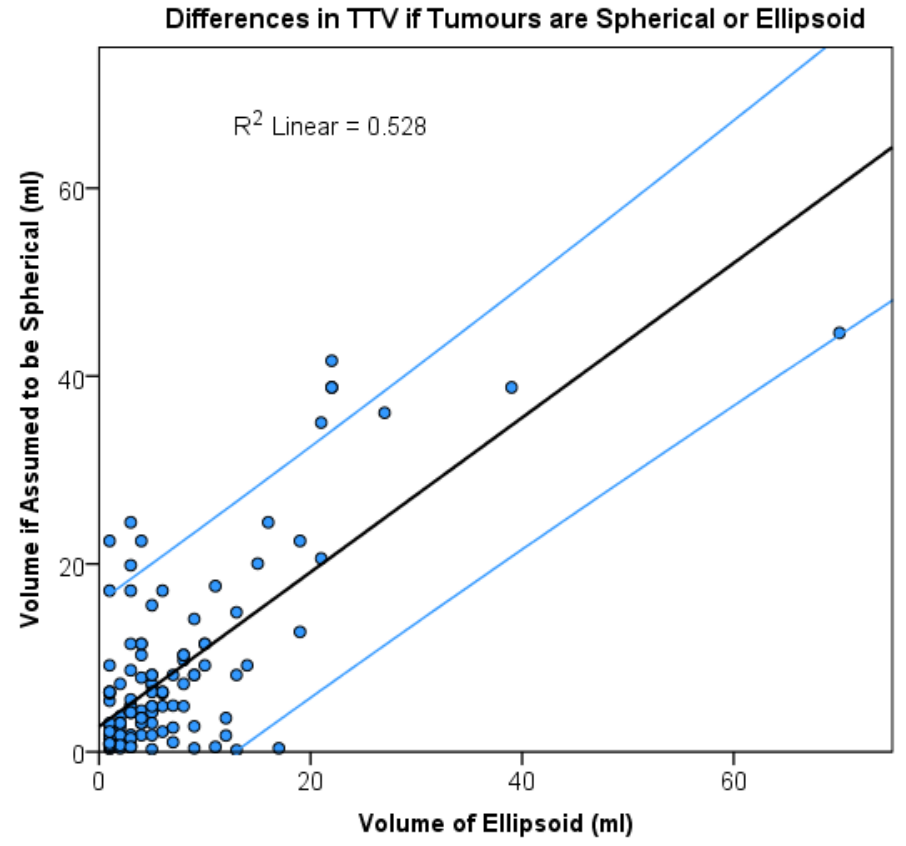

C

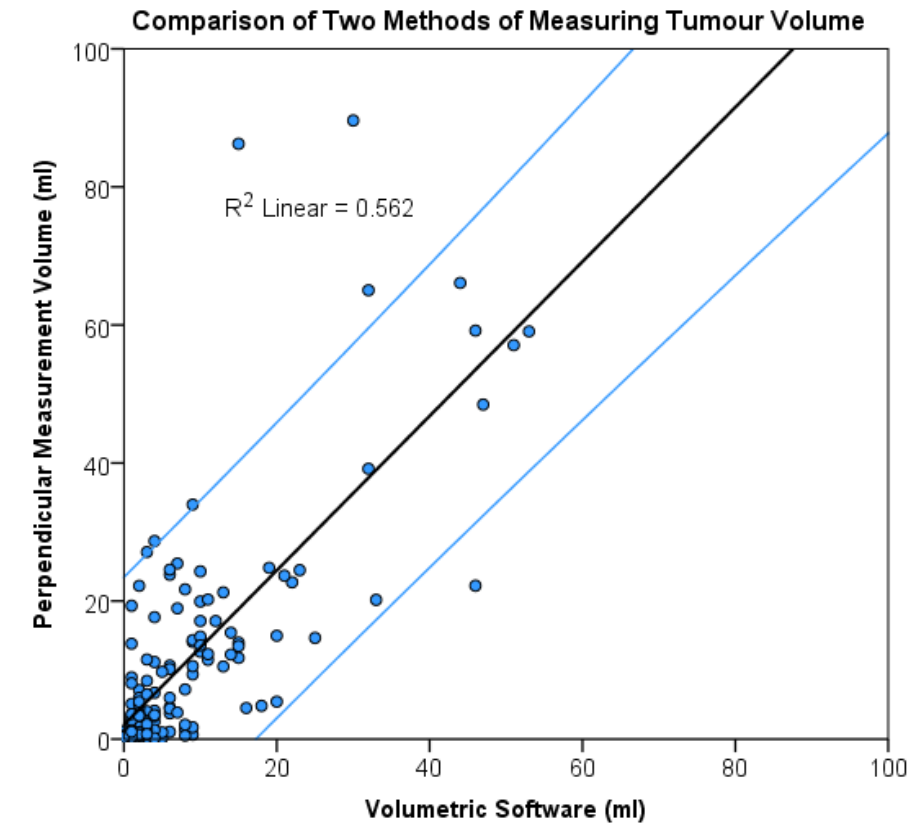




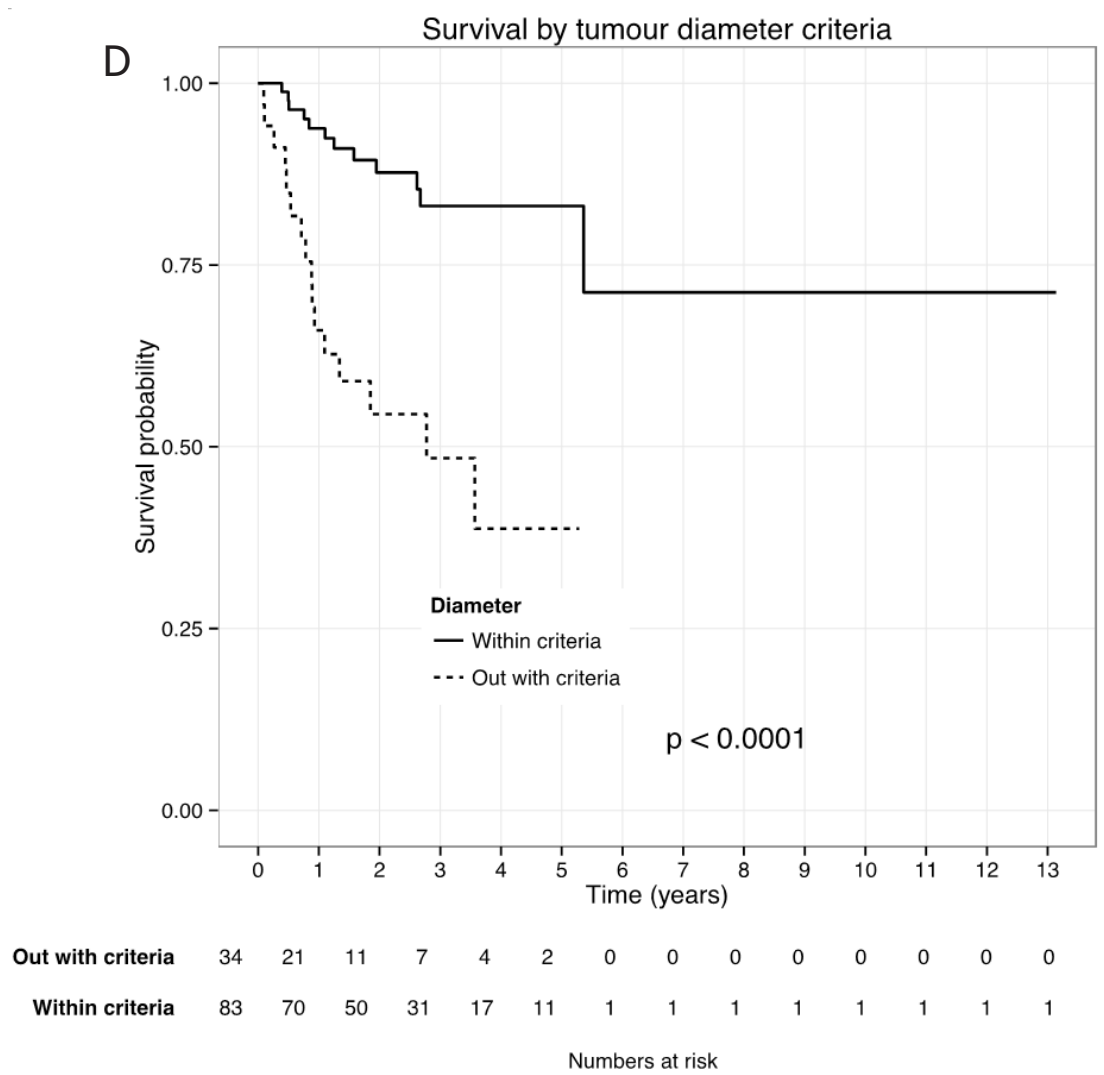

Figure 4. Example of an ellipsoid HCC (author's own image) (A); the difference in TTV if tumours are spherical or ellipsoid. Considering a single tumour nodule of $5 \mathrm{~cm}$ in diameter and varying the diameter to create an ellipsoid. Student's $t$-test was conducted showing a significant difference between the volume of ellipsoid versus the corresponding volume of the tumours, assumed to be spherical [t(123) $=-2.470, P=0.015](B)$; a comparison of the two methods of measuring tumour volume. Student's t-test showed that the mathematical equation method significantly overestimates tumour volume compared to the planimetry software $[t(141)=3.381, P=0.001]$ (C); overall survival of all patients with TTV within a theoretical volume-based Milan criteria. Patients then divided according to diameter-based criteria. Survival analysis was conducted to show a significant difference in survival between the two patient groups (log rank test chisquare $=21.4, P=0.0001)(D)$. HCC: hepatocellular carcinoma; TTV: total tumour volume

Table 2. Number and $\%$ of patients within/outside diameter/volume criteria

\begin{tabular}{lccc}
\hline Volume-based eligibility & \multicolumn{3}{c}{ Diameter-based eligibility } \\
\cline { 2 - 4 } & Within criteria & Outside criteria & Total \\
\hline Within criteria & $62(57 \%)$ & $41(38 \%)$ & $103(95 \%)$ \\
Outside criteria & $1(1 \%)$ & $5(5 \%)$ & $6(6 \%)$ \\
Total & $63(58 \%)$ & $46(42 \%)$ & 109 \\
\hline
\end{tabular}

The long-term outcome of patients based on their tumour diameter or TTV is beyond the remit of this study, but further research into this is required. However, similar studies have suggested that TTV may be a better predictor of outcome than diameter ${ }^{[16]}$. Ultimately, using volume to predict a patient's survival is unlikely to be optimal without allowing for other influential factors, such as comorbidities or functional hepatic reserve.

\section{DISCUSSION}

\section{Transplant eligibility criteria}

The Milan criteria are currently the standard for deciding the eligibility for OLT in HCC patients. Unfortunately, this approach has a number of limitations, including reliance on one tumour dimension - diameter, and is prone to inter-user variability ${ }^{[17]}$. Furthermore, the studies on which the Milan criteria 
are based were conducted during a period when the average OLT waiting time was $<6$ months ${ }^{[9]}$. With waiting times today often vastly exceeding this, the tumours which were originally within criteria are likely to have shown progression ${ }^{[9]}$. This may result in worse survival statistics than the Milan criteria predict. Many transplant centres have recognised that current criteria may be too strict and are now transplanting patients with larger tumours ${ }^{[16]}$. Although the Milan criteria place limits on the number of tumour nodules, some studies now suggest that excluding patients solely based on this is inaccurate ${ }^{[19]}$. In this study we investigated the effect of applying the Milan criteria to cover three tumour dimensions, with the current rules corresponding to a TTV $\leq 65.5 \mathrm{~mL}$ for one lesion and $\leq 42.4 \mathrm{~mL}$ for $<3$ lesions. However, as suggested by Figure 4, this cut-off value assumes tumours are spherical, and therefore, extrapolating volume from Milan criteria will tend to overestimate TTV. The outcome of patients with larger TTV has not yet been validated but has been suggested to correspond with poorer outcomes ${ }^{[2]}$.

\section{Measuring tumour diameters}

A limited number of studies have investigated the relative sensitivities and specificities of CT and MRI in the imaging of HCC. Most of these suggest that MRI is superior, especially in relation to small tumours, although CT is still more commonly used in practice ${ }^{[6,10,21]}$. However, these studies focus on the diagnosis of HCC and not the accuracy of measurements of the size of tumours. This study found no statistically significant difference in the measurements between CT and MRI. This justifies the lack of specification of imaging modalities in current guidelines. There are, however, very few studies comparing the sizes of tumours on CT and MRI to pathology reports. Some of these suggest that radiology overestimates tumour size, and others imply the opposite ${ }^{[22]}$. The current study demonstrated a fundamental flaw in measuring diameter at all - HCCs are not spherical. Imaging a tumour in one plane does not accurately represent the true shape and may, in fact, disadvantage patients whose tumour happens to be outside criteria based on transverse view alone. In this study, $94 \%$ of tumours studied were not spherical.

\section{Volume}

Some studies have identified tumour volume as an independent risk factor of HCC recurrence and as

a prognostic indicator ${ }^{[16,23]}$. One of these studies also found that if patients who were outside the Milan criteria but had a small TTV and underwent OLT, their survival was similar to those within the criteria ${ }^{[23]}$. Although, theoretically, measuring tumours in $3 \mathrm{D}$ increases the potential error from inter-user variation threefold, with the use of volumetric software, this error is greatly reduced ${ }^{[15]}$. Whilst initially a timeconsuming process, the author AG found that using the software became more rapid with practice. This study also found that the use of planimetry software is superior to using the mathematical equation for ellipsoid volume, as the latter does not allow for small fluctuation in the circumference of tumours. With advancing technology, it is likely that more automated 3D measurement software will become available, both decreasing the workload on individual and inter-user variability as a source of bias. This technology is already being trialled in other cancers such as breast ${ }^{[24]}$ and oropharyngeal ${ }^{[25]}$.

\section{Limitations of the study}

This study was based on a small sample size, made even smaller after allowance for incomplete data. For example, although there were 146 patients on the SLTU database who had undergone transplantation, complete imaging was only available for $61 \%$. However, most of these missing images were due to NHS Lothian changing the patient database software in 2007 and thus is unlikely to cause temporal systematic bias in the results. Over the 19-year period studied, a number of medical advances may have occurred, including improvements in imaging techniques. The authors did not notice this to a significant extent in the manual measurements on tumour volume. Another limitation of both this study and the Milan criteria was the lack of consideration of the underlying pathology of tumours, in terms of pathological differentiation and the underlying disease. No data were collected regarding any antitumour treatments (e.g., chemotherapy or radiotherapy); whilst this would not have implications on 3D measurements of 
initial tumours, it is reasonable to expect this may affect patient survival. The standard of care for HCC has changed dramatically over time. It is important to recognise the advances made in the treatment of HCC since this initial patient cohort was studied and future research should use a more contemporary data set. However, the criteria for transplantation remains based on tumour-load as measured in $2 \mathrm{D}$, our results suggest that $3 \mathrm{D}$ measurements may be beneficial in future updates to these criteria.

\section{Suggestions for future research}

There are many published studies investigating the survival implications of the Milan criteria and the effect of extending the rules. However, many of these studies neglect the effect of the shape and ultimately the volume of the tumour on survival. Ultimately, long-term prospective studies of patient groups with different total tumour volumes are required. This would allow for reliable information on the true impact of tumour volume on survival of patients. It would also be interesting to study a combined approach, using other factors including histology and genetic profile in combination with TTV, in selecting patients for transplantation. Ultimately, if revision of current guidelines is to be considered, validation of TTV and patient outcomes is required to select optimal cut-off values for tumour load.

In conclusion, This study showed that CT and MRI are comparable in terms of measuring the diameter of tumours, but that semi-automated planimetry software is superior to mathematical methods for calculating volume. The patients all had fairly similar sizes of tumours; however, the tumours were presumed to be spherical, and eligibility for transplant was based on a single dimension - diameter. In this study, we showed that owing to the ellipsoid shape of most tumours, measurement of volume captures the tumour size more accurately. The survival of patients ultimately depends upon OLT. Current reliance on diameter alone to determine eligibility limits the number of patients who may benefit from a donor liver. This study adds weight to the calls from other authors for revision of guidelines for transplantation.

\section{DECLARATIONS}

\section{Authors' contributions}

Conceived the study and wrote the manuscript: Graham AI, Wigmore SJ

Participated in the research contributed to the data and edited the manuscript: Adair A, Harrison EM, Gordon-Smith J, Ireland H, Patel D

\section{Availability of data and materials}

Not applicable.

\section{Financial support and sponsorship}

None.

\section{Conflicts of interest}

All authors declared that there are no conflicts of interest.

\section{Ethical approval and consent to participate}

Not applicable.

\section{Consent for publication}

Not applicable.

\section{Copyright}

(c) The Author(s) 2021. 


\section{REFERENCES}

1. Bray F, Ferlay J, Soerjomataram I, Siegel RL, Torre LA, Jemal A. Global cancer statistics 2018: GLOBOCAN estimates of incidence and mortality worldwide for 36 cancers in 185 countries. CA Cancer J Clin 2018;68:394-424.

2. Rawla P, Sunkara T, Muralidharan P, Raj JP. Update in global trends and aetiology of hepatocellular carcinoma. Contemp Oncol (Pozn) 2018;22:141-50.

3. Forner A, Llovet JM, Bruix J. Hepatocellular carcinoma. Lancet 2012;379:1245-55.

4. Bruix J, Llovet JM. Major achievements in hepatocellular carcinoma. Lancet 2009;373:614-6.

5. Ayuso C, Rimola J, García-Criado A. Imaging of HCC. Abdom Imaging 2012;37:215-30.

6. Choi BI, Lee JM. Advancement in HCC imaging: diagnosis, staging and treatment efficacy assessments: imaging diagnosis and staging of hepatocellular carcinoma. J Hepatobiliary Pancreat Sci 2010;17:369-73.

7. Llovet JM, Burroughs A, Bruix J. Hepatocellular carcinoma. Lancet 2003;362:1907-17.

8. Forner A, Reig M, Bruix J. Hepatocellular carcinoma. Lancet 2018;391:1301-14.

9. Befeler AS, Di Bisceglie AM. Hepatocellular carcinoma: diagnosis and treatment. Gastroenterology 2002;122:1609-19.

10. Llovet JM, Fuster J, Bruix J; Barcelona-Clínic Liver Cancer Group. The Barcelona approach: diagnosis, staging, and treatment of hepatocellular carcinoma. Liver Transpl 2004;10:S115-20.

11. Mazzaferro V, Regalia E, Doci R, et al. Liver transplantation for the treatment of small hepatocellular carcinomas in patients with cirrhosis. N Engl J Med 1996;334:693-9.

12. Duffy JP, Vardanian A, Benjamin E, et al. Liver transplantation criteria for hepatocellular carcinoma should be expanded: a 22-year experience with 467 patients at UCLA. Ann Surg 2007;246:502-9; discussion 509-11.

13. Therasse P, Arbuck SG, Eisenhauer EA, et al. New guidelines to evaluate the response to treatment in solid tumors. European Organization for Research and Treatment of Cancer, National Cancer Institute of the United States, National Cancer Institute of Canada. $J$ Natl Cancer Inst 2000;92:205-16.

14. Fleckenstein FN, Schernthaner RE, Duran R, et al. 3D Quantitative tumour burden analysis in patients with hepatocellular carcinoma before TACE: comparing single-lesion vs. multi-lesion imaging biomarkers as predictors of patient survival. Eur Radiol 2016;26:3243-52.

15. Prasad SR, Jhaveri KS, Saini S, Hahn PF, Halpern EF, Sumner JE. CT tumor measurement for therapeutic response assessment: comparison of unidimensional, bidimensional, and volumetric techniques initial observations. Radiology 2002;225:416-9.

16. Lee YH, Hsia CY, Hsu CY, Huang YH, Lin HC, Huo TI. Total tumor volume is a better marker of tumor burden in hepatocellular carcinoma defined by the Milan criteria. World J Surg 2013;37:1348-55.

17. Welsh JL, Bodeker K, Fallon E, Bhatia SK, Buatti JM, Cullen JJ. Comparison of response evaluation criteria in solid tumors with volumetric measurements for estimation of tumor burden in pancreatic adenocarcinoma and hepatocellular carcinoma. Am J Surg 2012;204:580-5.

18. Delis SG, Bakoyiannis A, Tassopoulos N, et al. Hepatic resection for hepatocellular carcinoma exceeding Milan criteria. Surg Oncol 2010;19:200-7.

19. Macaron C, Hanouneh IA, Lopez R, Aucejo F, Zein NN. Total tumor volume predicts recurrence of hepatocellular carcinoma after liver transplantation in patients beyond Milan or UCSF criteria. Transplant Proc 2010;42:4585-92.

20. Zakaria HM, Macshut M, Gaballa NK, et al. Total tumor volume as a prognostic value for survival following liver resection in patients with hepatocellular carcinoma. Retrospective cohort study. Ann Med Surg (Lond) 2020;54:47-53.

21. Lencioni R. Surveillance and early diagnosis of hepatocellular carcinoma. Dig Liver Dis 2010;42:S223-7.

22. Chen HY, Hou YL, Ma XM, Xie HY, Ye M, Bai YR. CT and MRI in target delineation in primary hepatocellular carcinoma. Cancer Radiother 2013;17:750-4.

23. Li C, Wen TF, Yan LN, et al. Scoring selection criteria including total tumour volume and pretransplant percentage of lymphocytes to predict recurrence of hepatocellular carcinoma after liver transplantation. PLoS One 2013;8:e72235.

24. Lagendijk M, Vos EL, Ramlakhan KP, et al. Breast and tumour volume measurements in breast cancer patients using 3-D automated breast volume scanner images. World J Surg 2018;42:2087-93.

25. Stuppner S, Waskiewicz J, Ruiu A. Gross tumour volume comparison in oropharynx carcinomas using different intelligent imaging software. A retrospective analysis. Pol J Radiol 2020;85:e287-92. 